\title{
DIFFERENTIAL IN INFRASTRUCTURE NETWORKS
}

\author{
Akin Kanli And Zeynep Nihan Odabaş BerberleR*
}

\begin{abstract}
Let $G=(V, E)$ be a graph of order $n$ and let $B(D)$ be the set of vertices in $V \backslash D$ that have a neighbor in the vertex set $D$. The differential of a vertex set $D$ is defined as $\partial(D)=|B(D)|-|D|$ and the maximum value of $\partial(D)$ for any subset $D$ of $V$ is the differential of $G$. A set $D$ of vertices of a graph $G$ is said to be a dominating set if every vertex in $V \backslash D$ is adjacent to a vertex in $D$. $G$ is a dominant differential graph if it contains a $\partial$-set which is also a dominating set. This paper is devoted to the computation of differential of wheel, cycle and path-related graphs as infrastructure networks. Furthermore, dominant differential wheel, cycle and path-related types of networks are recognized.
\end{abstract}

Mathematics Subject Classification. 05C69.

Received December 9, 2019. Accepted March 18, 2020.

\section{INTRODUCTION}

In this paper, simple, finite and undirected graphs without loops and multiple edges are considered. Let $G=(V, E)$ be a graph with vertex set $V$ and edge set $E$. The order of $G$ is given by $|V(G)|=n$ and the size is defined as $|E(G)|=m$ where $|*|$ denotes the number of elements in the set (i.e. the cardinality). The neighborhood of a vertex $v \in V(G)$ is the set of vertices adjacent to $v$, denoted $N_{G}(v)$ or just $N(v)$, and the closed neighborhood of $v$ is given by $N[v]=N(v) \cup\{v\}$. Thus, $N(v)=\{u \in V(G) \mid u v \in E(G)\}$ and $N(v)$ is referred to as the open neighborhood of $v$. The degree of a vertex $v \in V$ is defined as $d(v)=|N(v)|$. For a set $S \subseteq V, N(S)=\bigcup_{v \in S} N(V)$ and $N[S]=N(S) \cup S$. An end-vertex or a pendant or pendent vertex is a vertex of degree one and its neighbor is called a support vertex. For $S \subseteq V(G)$, the subgraph of $G$ induced by $S$ is denoted by $G[S]$. Let $G$ and $H$ be two disjoint graphs. The join of graphs $G$ and $H$, denoted by $G \vee H$, is obtained from the disjoint union $G$ and $H$ by adding the edges $\{x y \mid x \in V(G), y \in V(H)\}[21]$.

For any real number $x$ we define the ceiling function $\lceil x\rceil$ as the smallest integer greater than or equal to $x$ and similarly we define the floor function $\lfloor x\rfloor$ as the largest integer smallest than or equal to $x$.

Graph theoretic techniques provide a convenient tool for the investigation of networks. It is well-known that an interconnection network can be modeled by a graph with vertices representing sites of the network and edges representing links between sites of the network. Therefore various network problems can be studied by graph theoretical methods.

The differential in graphs is a subject of increasing interest, both in pure and applied mathematics. The research and application of the $\partial(G)$ appears mainly in computational mathematics. The differential of a graph

Keywords. Differential, domination, graph invariant.

Faculty of Science, Department of Computer Science, Dokuz Eylul University, Izmir 35160, Turkey.

*Corresponding author: zeynep. berberler@deu.edu.tr 
was introduced in [17] in 2006, and studied by several authors [1-9,14,16,19,20], motivated by its applications to information diffusion in social networks. The study of the mathematical properties of the differential in graphs stated in $[1-9,14,16,17,19,20]$. This parameter has been studied by many authors, both from the viewpoint of combinatorics and from the viewpoint of the algorithmic complexity. We refer to the papers $[1-9,14,16,17,19,20]$ and the literature quoted therein. Since computing the differential of a graph is NPcomplete in general, it becomes an interesting question to calculate differential for some special classes of interesting or practically useful graphs. In the following sections we will deal with this question.

Let $G=(V, E)$ be a graph of order $n$, for every set $D \subseteq V$ let $B(D)$ be the set of vertices in $V \backslash D$ that have a neighbor in the vertex set $D$. The differential of $D$ is defined as $\partial(D)=|B(D)|-|D|$ and the differential of a graph $G$, written $\partial(G)$, is equal to $\max \{\partial(D): D \subseteq V\}$. We will say that $D \subseteq V$ is a differential set or $\partial$-set if $\partial(D)=\partial(G)$ is called a $\partial$-set or differential set. Note that the connectivity of $G$ is not an important restriction, since if $G$ has connected components $G_{1}, \ldots, G_{k}$, then $\partial(G)=\partial\left(G_{1}\right)+\ldots+\partial\left(G_{k}\right)$. Therefore, we will only consider connected graphs.

A set $D$ of vertices of a graph $G$ is said to be a dominating set if every vertex in $V \backslash D$ is adjacent to a vertex in $D$. The domination number of $G$, denoted by $\gamma(G)$ is the minimum size of a dominating set of $G$ [21]. Research on domination in graphs has not only important theoretical signification, but also varied application in such fields as computer science, communication networks, ad hoc networks, biological and social networks, distributed computing, coding theory, and web graphs. Dominating sets in graphs are natural models for facility location problems in operations research. In general, the concept of dominating sets in graph theory finds wide applications in different types of communication networks. A broadcast from a communication vertex is received by all its neighbors. This is captured by the notion of domination in a graph. Finally, we will say that $G$ is a dominant differential graph if it contains a $\partial$-set which is also a dominating set. Some examples of dominant differential graphs are complete graphs, star graphs, wheel graphs, and path graphs $P_{n}$ and cycle graphs $C_{n}$ with $n=3 k$ or $n=3 k+2$.

The rest of the paper is structured as follows. In Section 2, the known results in literature are overviewed. In the following sections, the differential of wheel, cycle and path-related types of networks are computed and exact formulae are derived.

\section{KNOWN RESULTS}

Theorem 2.1 ([9]). The differential of

(a) the complete graph $K_{n}$ of order $n$ is $\partial\left(K_{n}\right)=n-2$;

(b) the path $P_{n}$ of order $n$ is $\partial\left(P_{n}\right)=\left\lfloor\frac{n}{3}\right\rfloor$;

(c) the cycle $C_{n}(n \geq 3)$ of order $n$ is $\partial\left(C_{n}\right)=\left\lfloor\frac{n}{3}\right\rfloor$;

(d) the star $K_{1, n}$ of order $n+1$ is $\partial\left(K_{1, n}\right)=n-1$;

(e) the complete bipartite graph $K_{m, n}$ of order $m+n$ is $\partial\left(K_{m, n}\right)=\max \{m-1, n-1, m+n-4\}$

(f) the wheel $W_{n}$ of order $n+1$ is $\partial\left(W_{n}\right)=n-1$.

Theorem 2.2 ([7]). A graph $G$ is dominant differential if and only if $\partial(G)=n-2 \gamma(G)$.

\section{Differential in WHeEL RELATED NeTWORKS}

In this section, the differential of wheel-related networks including gear and helm networks are calculated (Fig. 1).

\subsection{Gear networks}

Gear network is a wheel graph with a vertex added between each pair adjacent graph vertices of the outer cycle. $G_{n}$ has $2 n+1$ vertices and $3 n$ edges [12]. $G_{n}$ includes an even cycle $C_{2 n}$. There are two types of vertices 


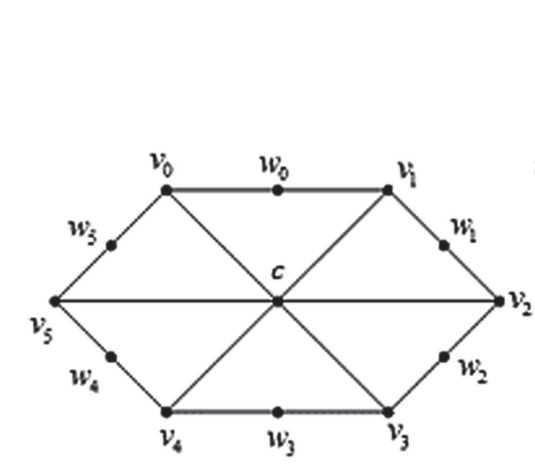

(a)

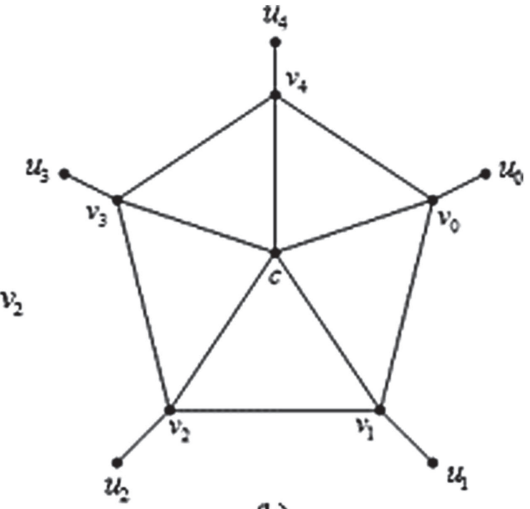

(b)

Figure 1. (a) Gear network $G_{n}$ for $n=6$. (b) Helm network $H_{n}$ for $n=5$.

of $C_{2 n}$ in $G_{n}$ as vertices of degree two and three, respectively. The vertices of degree two are referred to as minor vertices and vertices of degree three to as major vertices [15]. The central vertex $c$ of $G_{n}$ has degree of $n$. Label the major and minor vertices, respectively, as $v_{0}, \ldots, v_{n-1}$ and $w_{0}, \ldots, w_{n-1}$ and let $w_{i}$ be adjacent to the vertices $v_{i}$ and $v_{i+1}$ for $0 \leq i \leq n-1$, where $i+1$ is taken modulo $n$.

Theorem 3.1. The differential of the gear network $G_{n}$ of order $2 n+1$ is

$$
\partial\left(G_{n}\right)= \begin{cases}2+\left\lfloor\frac{2 n-3}{3}\right\rfloor, & \text { if } n=3 \\ n-1, & \text { if } n \geq 4\end{cases}
$$

Proof. If we take the central vertex $c$ and so $D_{1}=\{c\}$, then we have that $B\left\{D_{1}\right\}=\left\{v_{0}, \ldots, v_{n-1}\right\}$ and so $\partial\left(D_{1}\right)=n-1$, and taking any other subset of $V\left(G_{n}\right)$ to the set $D_{1}$ yields $\partial\left(D_{1}\right) \leq n-1$.

If we take a major vertex $v_{i}(0 \leq i \leq n-1)$ of $G_{n}$ to the set $D_{2}$, that is $D_{2}=\left\{v_{i}\right\}$, then we have $B\left(D_{2}\right)=\left\{c, w_{i-1}, w_{i}\right\}$, where $i-1$ is taken modulo $n$, yielding $\partial\left(D_{2}\right)=2$.

Let $S_{1}=V\left(G_{n}\right) \backslash N_{G_{n}}\left[v_{i}\right]$ and so we have that $G_{n}\left[S_{1}\right]=C_{2 n-3}$. If we take the maximal $\partial$-set of $G_{n}\left[S_{1}\right]$ to the set $D_{2}$ having the set $D_{3}$, then since $\partial\left(C_{n}\right)=\left\lfloor\frac{n}{3}\right\rfloor$, we receive $\partial\left(D_{3}\right)=\partial\left(D_{1}\right)+\partial\left(C_{2 n-3}\right)=2+\left\lfloor\frac{2 n-3}{3}\right\rfloor$, and taking any other subset of $V\left(G_{n}\right)$ to the set $D_{3}$ yields $\partial\left(D_{3}\right)<2+\left\lfloor\frac{2 n-3}{3}\right\rfloor$.

If we take a minor vertex $w_{i}(0 \leq i \leq n-1)$ of $G_{n}$ to the set $D_{4}$, that is $D_{4}=\left\{w_{i}\right\}$, then we have $B\left(D_{4}\right)=\left\{v_{i}, v_{i+1}\right\}$, where $i+1$ is taken modulo $n$, yielding $\partial\left(D_{4}\right)=1$.

Let $S_{2}=V\left(G_{n}\right) \backslash\left\{c, N_{G_{n}}\left[w_{i}\right]\right\}$ and so we have that $G_{n}\left[S_{2}\right]=C_{2 n-3}$. If we take the maximal $\partial$-set of $G_{n}\left[S_{2}\right]$ to the set $D_{4}$ having the set $D_{5}$, then since $\partial\left(C_{n}\right)=\left\lfloor\frac{n}{3}\right\rfloor$ and the maximal $\partial$-set of $G_{n}\left[S_{2}\right]$ includes at least one major vertex, we receive $\partial\left(D_{5}\right)=\partial\left(D_{4}\right)+\left(\partial\left(C_{2 n-3}\right)+1\right)=2+\left\lfloor\frac{2 n-3}{3}\right\rfloor$, and taking any other subset of $V\left(G_{n}\right)$ to the set $D_{5}$ yields $\partial\left(D_{5}\right)<2+\left\lfloor\frac{2 n-3}{3}\right\rfloor$.

By the definition of graph differential, among all the differential sets, we get

$$
\begin{aligned}
& \partial\left(G_{n}\right)=\max \left\{\partial\left(D_{k}\right)\right\}(1 \leq k \leq 5) \\
& \partial\left(G_{n}\right)= \begin{cases}2+\left\lfloor\frac{2 n-3}{3}\right\rfloor, & \text { if } n \leq 6 ; \\
n-1, & \text { if } n \geq 4 .\end{cases}
\end{aligned}
$$

Thus, the proof holds.

Remark 3.2. We can easily observe that $\gamma\left(G_{n}\right)=\left\lceil\frac{2 n}{3}\right\rceil$ and by Theorem 2.2 we conclude that gear networks are dominant differential for $n=3,4,6$. 


\subsection{Helm networks}

Helm $H_{n}$ is a network of order $2 n+1$ obtained from a wheel $W_{n}$ with cycle $C_{n}$ having a pendant edge attached to each vertex of the cycle. $H_{n}$ consists of the vertex set $V\left(H_{n}\right)=\left\{v_{i} \mid 0 \leq i \leq n-1\right\} \cup\left\{u_{i} \mid 0 \leq i \leq n-1\right\} \cup\{c\}$ and edge set $E\left(H_{n}\right)=\left\{v_{i} v_{i+1} \mid 0 \leq i \leq n-1\right\} \cup\left\{v_{i} u_{i} \mid 0 \leq i \leq n-1\right\} \cup\left\{v_{i} c \mid 0 \leq i \leq n-1\right\}$, where $i+1$ is taken modulo $n$ [12]. The central vertex $c$ of $H_{n}$ has a vertex degree of $n$. There are two types of vertices in $H_{n} \backslash\{c\}$ as the vertices of degree four and one, respectively. The vertices of degree one and four are referred to as minor and major vertices, respectively [15].

Theorem 3.3. The differential of the helm network $H_{n}$ of order $2 n+1$ is

$$
\partial\left(H_{n}\right)= \begin{cases}3+2\left\lfloor\frac{n-3}{3}\right\rfloor, & \text { if } n=3 \\ n-1, & \text { if } n \geq 4\end{cases}
$$

Proof. If we take the central vertex $c$ of $H_{n}$ to the set $D_{1}$, then we have $B\left\{D_{1}\right\}=\left\{v_{0}, \ldots, v_{n-1}\right\}$ yielding $\partial\left(D_{1}\right)=n-1$, and taking any other subset of $V\left(H_{n}\right)$ to the set $D_{1}$ yields $\partial\left(D_{1}\right)<n-1$.

If we take a major vertex $v_{i}(0 \leq i \leq n-1)$ of $H_{n}$ to the set $D_{2}$, that is $D_{2}=\left\{v_{i}\right\}$, then we have $B\left(D_{2}\right)=\left\{c, u_{i}, v_{i+1}, v_{i-1}\right\}$, where $i+1$ and $i-1$ are taken modulo $n$, yielding $\partial\left(D_{2}\right)=3$.

Let $S_{1}=V\left(H_{n}\right) \backslash N_{H_{n}}\left[v_{i}\right]$ and so we have that $H_{n}\left[S_{1}\right]=P_{n-3}^{*}$ where $P_{n}^{*}$ is the path graph of order $n$ with a pendant vertex attached to each vertex of the path. If we take the maximal $\partial$-set of $P_{n-3}$ to the set $D_{2}$ having the set $D_{3}$, then since $\partial\left(P_{n}\right)=\left\lfloor\frac{n}{3}\right\rfloor$ and every vertex of $P_{n-3}$ is adjacent to a pendant vertex, we receive

$$
\begin{aligned}
& \partial\left(D_{3}\right)= \begin{cases}\partial\left(D_{2}\right)+\partial\left(P_{n-3}\right)+\left\lfloor\frac{n-3}{3}\right\rfloor, & \text { if } n=3 k \text { or } n=3 k+1 ; \\
\partial\left(D_{2}\right)+\partial\left(P_{n-3}\right)+\left\lceil\frac{n-3}{3}\right\rceil, & \text { if } n=3 k+2,\end{cases} \\
& \partial\left(D_{3}\right)= \begin{cases}3+2\left\lfloor\frac{n-3}{3}\right\rfloor, & \text { if } n=3 k \text { or } n=3 k+1 ; \\
3+\left\lfloor\frac{n-3}{3}\right\rfloor+\left\lceil\frac{n-3}{3}\right\rceil, & \text { if } n=3 k+2,\end{cases}
\end{aligned}
$$

where $k \in \mathbb{Z}$ and taking any other subset of $V\left(H_{n}\right)$ to the set $D_{3}$, yields

$$
\partial\left(D_{3}\right)< \begin{cases}3+2\left\lfloor\frac{n-3}{3}\right\rfloor, & \text { if } n=3 k \text { or } n=3 k+1 \\ 3+\left\lfloor\frac{n-3}{3}\right\rfloor+\left\lceil\frac{n-3}{3}\right\rceil, & \text { if } n=3 k+2 .\end{cases}
$$

If we take a minor vertex $u_{i}(0 \leq i \leq n-1)$ of $H_{n}$ to the set $D_{4}$, then we have $B\left(D_{4}\right)=\left\{v_{i}\right\}$, yielding $\partial\left(D_{4}\right)=0$.

Let $S_{2}=V\left(H_{n}\right) \backslash N_{H_{n}}\left[u_{i}\right]$ and so we have the graph $H_{n}\left[S_{2}\right]$ including the central vertex $c, n-1$ major and $n-1$ minor vertices of $H_{n}$, and also $n-1$ major vertices induce the subgraph $P_{n-1}$ in $H_{n}\left[S_{2}\right]$. If we take the maximal $\partial$-set of $P_{n-1}$ to the set $D_{4}$ having the set $D_{5}$, then since $\partial\left(P_{n}\right)=\left\lfloor\frac{n}{3}\right\rfloor$ and every major vertex is adjacent to a pendant vertex and the central vertex $c$, we receive

$$
\begin{aligned}
& \partial\left(D_{5}\right)= \begin{cases}\partial\left(D_{4}\right)+\partial\left(P_{n-1}\right)+1+\left\lfloor\frac{n-1}{3}\right\rfloor, & \text { if } n=3 k+1 \text { or } n=3 k+2 ; \\
\partial\left(D_{4}\right)+\partial\left(P_{n-1}\right)+1+\left\lceil\frac{n-1}{3}\right\rceil, & \text { if } n=3 k,\end{cases} \\
& \partial\left(D_{5}\right)= \begin{cases}2\left\lfloor\frac{n-1}{3}\right\rfloor+1, & \text { if } n=3 k+1 \text { or } n=3 k+2 ; \\
\left\lfloor\frac{n-1}{3}\right\rfloor+\left\lceil\frac{n-1}{3}\right\rceil, & \text { if } n=3 k,\end{cases}
\end{aligned}
$$

where $k \in \mathbb{Z}$ and taking any other subset of $V\left(H_{n}\right)$ to the set $D_{5}$ yields

$$
\partial\left(D_{5}\right)< \begin{cases}2\left\lfloor\frac{n-1}{3}\right\rfloor+1, & \text { if } n=3 k+1 \text { or } n=3 k+2 ; \\ \left\lfloor\frac{n-1}{3}\right\rfloor+\left\lceil\frac{n-1}{3}\right\rceil+1, & \text { if } n=3 k .\end{cases}
$$




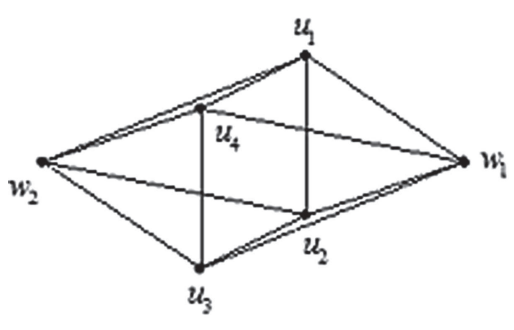

(a)

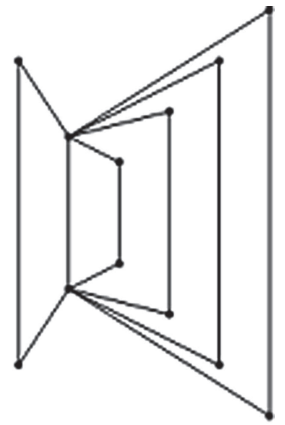

(b)

Figure 2. (a) Bipyramid network $\mathrm{BP}(n)$. (b) $n$-gon book of $k$-pages network $B(n, k)$ for $n=4, n=5$.

By the definition of graph differential, among all the differential sets, we get

$$
\begin{aligned}
& \partial\left(H_{n}\right)=\max \left\{\partial\left(D_{l}\right)\right\}(1 \leq l \leq 5) \\
& \partial\left(H_{n}\right)= \begin{cases}3+2\left\lfloor\frac{n-3}{3}\right\rfloor, & \text { if } n=3 ; \\
n-1, & \text { if } n \geq 4 .\end{cases}
\end{aligned}
$$

Thus, the proof holds.

\section{Differential in CYCLE RELATED NETWORKS}

In this section, the differential of cycle-related graphs including $k$-pyramid and $n$-gon book of $k$-pages networks are calculated (Fig. 2).

\section{1. $k$-pyramids}

The join graph $C_{n} \vee N_{k}(n \geq 3, k \geq 1)$, where $N_{k}$ is the null graph of order $k$, is called $k$-pyramid and is denoted by $k P(n)$. The 2-pyramid network $C_{n} \vee N_{2}$ is called bipyramid network and is denoted by $\operatorname{BP}(n)$. The 1-pyramid network $C_{n} \vee N_{1}$ is the wheel graph $W_{n}$ [12].

Let $u_{1}, u_{2}, u_{3}, \ldots, u_{n}$ be the vertices of $C_{n}$ and $w_{1}, w_{2}, w_{3}, \ldots, w_{k}$ be the vertices of $N_{k}$. Then, we have $\operatorname{deg}\left(u_{i}\right)=k+2(1 \leq i \leq n)$ and $\operatorname{deg}\left(w_{j}\right)=n(1 \leq j \leq k)$.

Theorem 4.1. The differential of the k-pyramid network $k P(n)$ of order $n+k$ is

$$
\partial(k P(n))= \begin{cases}n-1, \quad\left\{\begin{array}{l}
\text { if } n=3 t \quad \text { and } n \geq \frac{3 k+3}{2} \\
\text { if } n=3 t+1 \text { and } n \geq \frac{3 k+2}{2} \\
\text { if } n=3 t+2 \text { and } n \geq \frac{3 k+1}{2}
\end{array}\right. \\
\left\lfloor\frac{n}{3}\right\rfloor+k, \text { otherwise, }\end{cases}
$$

where $t \in \mathbb{Z}$.

Proof. If we take a vertex $w_{j}(1 \leq j \leq k)$ of $N_{k}$ in $k P(n)$ to the set $D_{1}$, that is $D_{1}=\left\{w_{j}\right\}$, then we have $B\left(D_{1}\right)=\left\{u_{1}, \ldots, u_{n}\right\}$ and so $\partial\left(D_{1}\right)=n-1$, and taking any other subset of $V(k P(n))$ to the set $D_{1}$ yields $\partial\left(D_{1}\right)<n-1$.

If we take the maximal $\partial$-set of $C_{n}$ in $k P(n)$ to the set $D_{2}$, then since $\partial\left(C_{n}\right)=\left\lfloor\frac{n}{3}\right\rfloor$ and every vertex $u_{i}(1 \leq i \leq n)$ of $C_{n}$ in $k P(n)$ is adjacent to every vertex $w_{j}(1 \leq j \leq k)$ of $N_{k}$, we have $\partial\left(D_{2}\right)=\left\lfloor\frac{n}{3}\right\rfloor+k$, and taking any other subset of $V(k P(n))$ to the set $D_{2}$ yields $\partial\left(D_{2}\right)<\left\lfloor\frac{n}{3}\right\rfloor+k$. 
By the definition of graph differential, we have

$$
\begin{aligned}
& \partial(k P(n))=\max \left\{\partial\left(D_{p}\right)\right\} \quad(p=1,2) \\
& \partial(k P(n))= \begin{cases}n-1, & \left\{\begin{array}{l}
\text { if } n=3 t \quad \text { and } n \geq \frac{3 k+3}{2} \\
\text { if } n=3 t+1 \text { and } n \geq \frac{3 k+2}{2} \\
\text { if } n=3 t+2 \text { and } n \geq \frac{3 k+1}{2}
\end{array}\right. \\
\left\lfloor\frac{n}{3}\right\rfloor+k, \text { otherwise }\end{cases}
\end{aligned}
$$

where $t \in \mathbb{Z}$. Thus, the proof holds.

Corollary 4.2. The differential of the bipyramid network $\mathrm{BP}(n)$ of order $n+2$ is

$$
\partial(\operatorname{BP}(n))= \begin{cases}\left\lfloor\frac{n}{3}\right\rfloor+2, & \text { if } n=3 \\ n-1, & \text { if } n>3 .\end{cases}
$$

Remark 4.3. Since the wheel network $W_{n}$ is 1 -pyramid network $C_{n} \vee N_{1}$, by taking $k=1$, the value of $\partial\left(W_{n}\right)$ in Theorem 2.1(f) holds.

Remark 4.4. We can easily observe that $\gamma(k P(n))=k$ and by Theorem 2.2 we conclude that $k$-pyramids are dominant differential networks for

$$
\left\{\begin{array}{l}
n=3 t \quad \text { and } n=3 k \\
n=3 t+1 \text { and } n=\frac{6 k-1}{2} \\
n=3 t+2 \text { and } n=3 k-1
\end{array}\right.
$$

where $t \in \mathbb{Z}$.

\section{2. $n$-gon books}

When $k$ copies of $C_{n}(n \geq 3)$ share a common edge, it will form an $n$-gon book of $k$ pages and is denoted by $B(n, k)$. The degree set of $B(n, k)$ is $\{2, k+1\}$ [12]. Therefore, the vertices of $B(n, k)$ are of two kinds: vertices of degree 2 are referred to as minor vertices and vertices of degree $k+1$ to as major vertices. For $k=1$, we notice that $B(n, k) \cong C_{n}$.

Theorem 4.5. The differential of the $n$-gon book of $k(k>1)$ pages network $B(n, k)$ of order $(n-2) k+2$ is

$$
\partial(B(n, k))= \begin{cases}\frac{n k}{3}, & \text { if } n=3 t \\ k\left(\left\lfloor\frac{n-4}{3}\right\rfloor+2\right)-2, & \text { if } n \neq 3 t\end{cases}
$$

where $t \in \mathbb{Z}$.

Proof. If we take one of the major vertices of $B(n, k)$-say vertex $u$, to the set $D_{1}$, that is $D_{1}=\{u\}$, then since $\left|N_{B(n, k)}(u)\right|=k+1$, we have $\partial\left(D_{1}\right)=k$.

Let $S_{1}=V(B(n, k)) \backslash N_{B(n, k)}[u]$ and so we have that $B(n, k)\left[S_{1}\right]=\bigcup_{i=1}^{k} P_{n-3}$. If we take the maximal $\partial$-set of $B(n, k)\left[S_{1}\right]$ to the set $D_{1}$ having the set $D_{2}$, then since $\partial\left(P_{n}\right)=\left\lfloor\frac{n}{3}\right\rfloor$, we have $\partial\left(D_{2}\right)=k\left\lfloor\frac{n-3}{3}\right\rfloor+k$.

For $n=3 t+1(t \in \mathbb{Z})$, if we take the other major vertex of $B(n, k)$-say vertex $v$ to the set $D_{2}$ having the set $D_{3}$, then we have $\partial\left(D_{3}\right) \leq\left(k\left\lfloor\frac{n-3}{3}\right\rfloor+k\right)+(k-2)=k\left\lfloor\frac{n-3}{3}\right\rfloor+2 k-2$, and taking any other subset of $V(B(n, k))$ to the set $D_{3}$ yields $\partial\left(D_{3}\right)<k\left\lfloor\frac{n-3}{3}\right\rfloor+2 k-2$.

If we take $D_{4}=\{u, v\}$ as the set of major vertices, then we have $\partial\left(D_{4}\right)=2 k-2$.

For $n \neq 3 t+1(t \in \mathbb{Z})$, let $S_{2}=V(B(n, k)) \backslash N_{B(n, k)}\left[D_{3}\right]$ and so we have that $B(n, k)\left[S_{2}\right]=\bigcup_{i=1}^{k} P_{n-4}$. If we take the maximal $\partial$-set of $B(n, k)\left[S_{2}\right]$ to the set $D_{4}$ having the set $D_{5}$, then since $\partial\left(P_{n}\right)=\left\lfloor\frac{n}{3}\right\rfloor$, we have $\partial\left(D_{5}\right)=2 k-2+k\left\lfloor\frac{n-4}{3}\right\rfloor$, and taking any other subset of $V(B(n, k))$ yields $\partial\left(D_{5}\right)<2 k-2+k\left\lfloor\frac{n-4}{3}\right\rfloor$. 


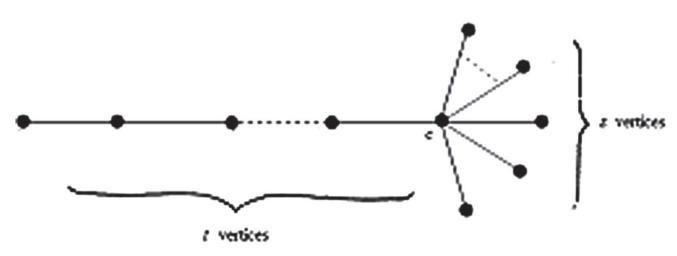

(a)

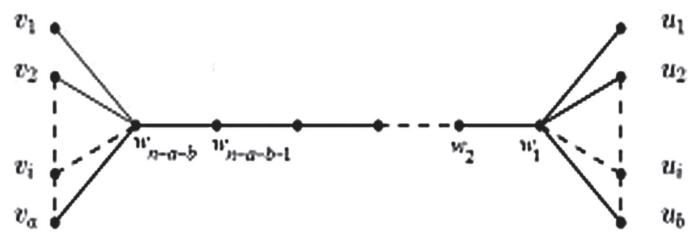

(b)

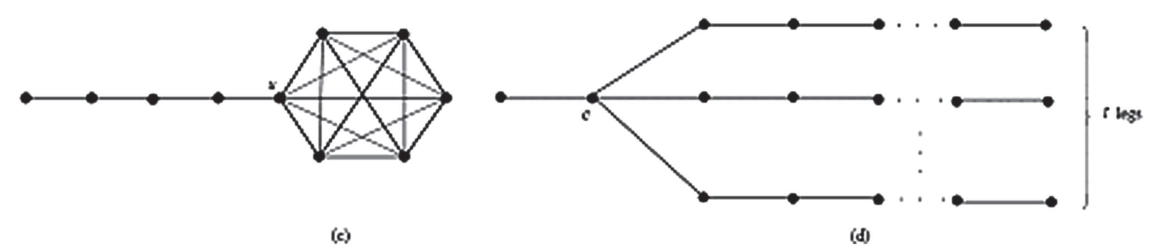

Figure 3. (a) Comet network $C_{s, t}$. (b) Double comet network DC $(n, a, b)$. (c) Lollipop networks $L_{n, d}$ for $n=10$ and $d=5$. (d) $E_{p}^{t}$ network.

By the definition of graph differential, among the differential sets, we receive for $k>1$

$$
\begin{aligned}
& \partial(B(n, k))=\max \left\{\partial\left(D_{i}\right)\right\} \quad(1 \leq i \leq 5) \\
& \partial(B(n, k))= \begin{cases}k\left(\left\lfloor\frac{n-3}{3}\right\rfloor+1\right), & \text { if } n=3 t ; \\
k\left(\left\lfloor\frac{n-4}{3}\right\rfloor+2\right)-2, & \text { if } n \neq 3 t,\end{cases}
\end{aligned}
$$

where $t \in \mathbb{Z}$. The theorem is thus proved.

Remark 4.6. We can easily observe that

$$
\gamma(B(n, k))= \begin{cases}1+k\left\lceil\frac{n-3}{3}\right\rceil, & \text { if } n=3 t \text { or } n=3 t+2 \\ 2+k\left\lceil\frac{n-4}{3}\right\rceil, & \text { if } n=3 t+1,\end{cases}
$$

where $t \in \mathbb{Z}$ and by Theorem 2.2 we conclude that $n$-gon books are dominant differential networks for $n=3 t+2$ and $k=2$ or $n=3 t(t \in \mathbb{Z})$.

\section{Differential in PATH RELATED NetWORKS}

In this section, the differential of path-related networks including comet, double comet, lollipop and $E_{p}^{t}$ networks are calculated (Fig. 3).

\subsection{Comet networks}

The comet $C_{s, t}$ where $s$ and $\mathrm{t}$ are positive integers, denotes the tree obtained by identifying the center of the star $K_{1, s}$ with an end-vertex of $P_{t}$, the path of order $t$. So $C_{s, 1} \cong K_{1, s}$ and $C_{1, p-1} \cong P_{p}$ [13]. Let the center of the star $K_{1, s}$-that is one end-vertex of $P_{t}$ be the vertex $c$.

Theorem 5.1. The differential of the comet network $C_{s, t}$ of order $s+t$ is

$$
\partial\left(C_{s, t}\right)=s+\left\lfloor\frac{t-2}{3}\right\rfloor .
$$


Proof. If we take the center vertex $c$ of $K_{1, s}$ in $C_{s, t}$ to the set $D_{1}$, that is $D_{1}=\{c\}$, then we receive $\partial\left(D_{1}\right)=5$.

Let $S_{1}=V\left(C_{s, t}\right) \backslash N_{C_{s, t}}[c]$ and so we have that $C_{s, t}\left[S_{1}\right]=P_{t-2}$. If we take the maximal $\partial$-set of $C_{s, t}\left[S_{1}\right]$ to the set $D_{1}$ having the set $D_{2}$, then since $\partial\left(P_{n}\right)=\left\lfloor\frac{n}{3}\right\rfloor$, we have $\partial\left(D_{2}\right)=s+\left\lfloor\frac{t-2}{3}\right\rfloor$, and taking any other subset of $V\left(C_{s, t}\right)$ to the set $D_{2}$ yields $\partial\left(D_{2}\right)<s+\left\lfloor\frac{t-2}{3}\right\rfloor$.

If we take one of the vertices of $K_{1, s}$ except the center vertex $c$ in $C_{s, t}$ to the set $D_{3}$, then we have $\partial\left(D_{3}\right)=0$.

Let $S_{2}=V\left(C_{s, t}\right) \backslash N_{C_{s, t}}\left[D_{3}\right]$ and so we have that $C_{s, t}\left[S_{2}\right]=P_{t-1}$. If we take the maximal $\partial$-set of $C_{s, t}\left[S_{2}\right]$ to the set $D_{3}$ having the set $D_{4}$, then since $\partial\left(P_{n}\right)=\left\lfloor\frac{n}{3}\right\rfloor$, we have $\partial\left(D_{4}\right)=\left\lfloor\frac{t-1}{3}\right\rfloor$, and taking any other subset of $V\left(C_{s, t}\right)$ to the set $D_{4}$ yields $\partial\left(D_{4}\right)<\left\lfloor\frac{t-1}{3}\right\rfloor$.

If we take the maximal $\partial$-set of $P_{t}$ to the set $D_{5}$ such that $c \in B\left(D_{5}\right)$, then since $\partial\left(P_{n}\right)=\left\lfloor\frac{n}{3}\right\rfloor$, we have $\partial\left(D_{5}\right)=\left\lfloor\frac{t}{3}\right\rfloor$.

If we take the center vertex $c$ of $K_{1, s}$ in $C_{s, t}$ to the set $D_{5}$ having the set $D_{6}$, then we have $\partial\left(D_{6}\right)=\left\lfloor\frac{t}{3}\right\rfloor+s-2$, and taking any other subset of $V\left(C_{s, t}\right)$ to the set $D_{6}$ yields $\partial\left(D_{6}\right)<\left\lfloor\frac{t}{3}\right\rfloor+s-2$.

By the definition of graph differential, we get

$$
\begin{aligned}
& \partial\left(C_{s, t}\right)=\max \left\{\partial\left(D_{i}\right)\right\} \quad(1 \leq i \leq 6) \\
& \partial\left(C_{s, t}\right)=s+\left\lfloor\frac{t-2}{3}\right\rfloor
\end{aligned}
$$

Thus the proof holds.

Remark 5.2. We can easily observe that $\gamma\left(C_{s, t}\right)=1+\left\lceil\frac{t-2}{3}\right\rceil$ and by Theorem 2.2 we conclude that comets are dominant differential networks for $t=3 k+2$ or $t=3 k+4(k \in \mathbb{Z})$.

\subsection{Double comet networks}

For $a, b>1, n \geq a+b+2$ by $\operatorname{DC}(n, a, b)$ we denote a double comet, which is a tree composed of a path containing $n-a-b$ vertices with $a$ pendent vertices attached to one of the ends of the path and $b$ pendent vertices attached to the other end of the path. Thus, $\operatorname{DC}(n, a, b)$ has $n$ vertices and $a+b$ leaves [11]. Let $v_{1}, v_{2}, \ldots, v_{a}, u_{1}, u_{2}, \ldots, u_{b}, w_{1}, w_{2}, \ldots, w_{n-a-b}$ be the vertex set of the double comet $\mathrm{DC}(n, a, b)$, which is obtained from a path $P_{n-a-b}$ of vertices $w_{1}, w_{2}, \ldots, w_{n-a-b}$ by attaching the pendant vertices $u_{1}, u_{2}, \ldots, u_{b}$ to the one end vertex $w_{1}$ of $P_{n-a-b}$ and attaching the pendant vertices $v_{1}, v_{2}, \ldots, v_{a}$ to the other end vertex $w_{n}$ of $P_{n-a-b}$.

Theorem 5.3. The differential of the double comet network $\operatorname{DC}(n, a, b)$ of order $n(a, b>1, n-a-b>2)$ is

$$
\partial(\mathrm{DC}(n, a, b))=a+b+\left\lfloor\frac{n-a-b-4}{3}\right\rfloor .
$$

Proof. If we take the vertex $w_{1}$ of $\mathrm{DC}(n, a, b)$ to the set $D_{1}$, that is $\partial\left(D_{1}\right)=a$.

Let $S_{1}=V(\mathrm{DC}(n, a, b)) \backslash N_{\mathrm{DC}(n, a, b)}\left[w_{1}\right]$ and so we have that $\operatorname{DC}(n, a, b)\left[S_{1}\right]=C_{b, n-a-b-2}$, where $C_{b, n-a-b-2}$ is a comet graph of order $n-a-2$. If we take the maximal $\partial$-set of $\mathrm{DC}(n, a, b)\left[S_{1}\right]$ to the set $D_{1}$ having the set $D_{2}$, then since $\partial\left(C_{s, t}\right)=s+\left\lfloor\frac{t-2}{3}\right\rfloor$, we receive $\partial\left(D_{2}\right)=a+b+\left\lfloor\frac{n-a-b-4}{3}\right\rfloor$, and taking any other subset of $V(\operatorname{DC}(n, a, b))$ to the set $D_{2}$ yields $\partial\left(D_{2}\right)<a+b+\left\lfloor\frac{n-a-b-4}{3}\right\rfloor$.

If we take the vertex $w_{n-a-b}$ of $\operatorname{DC}(n, a, b)$ to the set $D_{3}$, then we have $\partial\left(D_{3}\right)=b$.

Let $S_{2}=V(\mathrm{DC}(n, a, b)) \backslash N_{\mathrm{DC}(n, a, b)}\left[w_{n}\right]$ and so we have that $\operatorname{DC}(n, a, b)\left[S_{2}\right]=C_{a, n-a-b-2}$. If we take the maximal $\partial$-set of $\operatorname{DC}(n, a, b)\left[S_{2}\right\rfloor$ to the set $D_{3}$ having the set $D_{4}$, then since $\partial\left(C_{s, t}\right)=s+\left\lfloor\frac{t-2}{3}\right\rfloor$, we have $\partial\left(D_{4}\right)=b+a+\left\lfloor\frac{n-a-b-4}{3}\right\rfloor$, and taking any other subset of $V(\operatorname{DC}(n, a, b))$ to the set $D_{4}$ yields $\partial\left(D_{4}\right)<b+a+\left\lfloor\frac{n-a-b-4}{3}\right\rfloor$.

If we take the vertex $v_{i}(1 \leq i \leq a)$ of $\mathrm{DC}(n, a, b)$ to the set $D_{5}$, then we have $\partial\left(D_{5}\right)=0$. 
Let $S_{3}=V(\mathrm{DC}(n, a, b)) \backslash N_{\mathrm{DC}(n, a, b)}\left[v_{i}\right]$ and so we have that $\mathrm{DC}(n, a, b)\left[S_{3}\right]=\overline{K_{a-1}} \cup C_{b, n-a-b-1}$. If we take the maximal $\partial$-set of $\operatorname{DC}(n, a, b)\left[S_{3}\right]$ to the set $D_{5}$ having the set $D_{6}$, then since $\partial\left(C_{s, t}\right)=s+\left\lfloor\frac{t-2}{3}\right\rfloor$, we have $\partial\left(D_{6}\right)=\partial\left(C_{b, n-a-b-1}\right)=b+\left\lfloor\frac{n-a-b-3}{3}\right\rfloor$, and taking any other subset of $V(\operatorname{DC}(n, a, b))$ to the set $D_{6}$ yields $\partial\left(D_{6}\right)<b+\left\lfloor\frac{n-a-b-3}{3}\right\rfloor$.

If we take the vertex $u_{j}(1 \leq j \leq b)$ of $\mathrm{DC}(n, a, b)$ to the set $D_{7}$, then we have $\partial\left(D_{7}\right)=0$.

Let $S_{4}=V(\operatorname{DC}(n, a, b)) \backslash N_{\mathrm{DC}(n, a, b)}\left[u_{j}\right]$ and so we have that $\mathrm{DC}(n, a, b)\left[S_{4}\right]=\overline{K_{b-1}} \cup C_{a, n-a-b-1}$. If we take the maximal $\partial$-set of $\operatorname{DC}(n, a, b)\left[S_{4}\right]$ to the set $D_{7}$ having the set $D_{8}$, then since $\partial\left(C_{s, t}\right)=s+\left\lfloor\frac{t-2}{3}\right\rfloor$, we have $\partial\left(D_{8}\right)=\partial\left(C_{a, n-a-b-1}\right)=a+\left\lfloor\frac{n-a-b-3}{3}\right\rfloor$, and taking any other subset of $V(\operatorname{DC}(n, a, b))$ to the set $D_{8}$ yields $\partial\left(D_{8}\right)<a+\left\lfloor\frac{n-a-b-3}{3}\right\rfloor$.

For $n-a-b=3 k+1(k \in \mathbb{Z})$, if we take the maximal $\partial$-set of $P_{n-a-b}$ to the set $D_{9}$ such that $w_{1} \in B\left(D_{9}\right)$ or $w_{n-a-b} \in B\left(D_{9}\right)$, then since $\partial\left(P_{n}\right)=\left\lfloor\frac{n}{3}\right\rfloor$, we have $\partial\left(D_{9}\right)=\left\lfloor\frac{n-a-b}{3}\right\rfloor$. If $w_{1} \in B\left(D_{9}\right)$, then let $S_{5}=V(\mathrm{DC}(n, a, b)) \backslash N_{\mathrm{DC}(n, a, b)}\left[D_{9} \cup B\left(D_{9}\right)\right]$ and so we have $\operatorname{DC}(n, a, b)\left[S_{5}\right]=\overline{K_{b}} \cup K_{1, a}$. If we take the maximal $\partial$-set of $\operatorname{DC}(n, a, b)\left[S_{5}\right]$ to the set $D_{9}$ having the set $D_{10}$, then since $\partial\left(K_{1, n}\right)=n-1$, we have $\partial\left(D_{10}\right)=\left\lfloor\frac{n-a-b}{3}\right\rfloor+(a-1)$. If we take the vertex $w_{1}$ of $\mathrm{DC}(n, a, b)$ to the set $D_{10}$ having the set $D_{11}$, we have $\partial\left(D_{11}\right)=\left\lfloor\frac{n-a-b}{3}\right\rfloor+(a+b-3)$, and taking any other subset of $V(\operatorname{DC}(n, a, b))$ to the set $D_{11}$ yields $\partial\left(D_{11}\right)<\left\lfloor\frac{n-a-b}{3}\right\rfloor+(a+b-3)$. If $w_{n-a-b} \in B\left(D_{9}\right)$, then let $S_{6}=V(\operatorname{DC}(n, a, b)) \backslash N_{\mathrm{DC}(n, a, b)}\left[D_{9} \cup B\left(D_{9}\right)\right]$ and so we have $\operatorname{DC}(n, a, b)\left[S_{6}\right]=\overline{K_{a}} \cup K_{1, b}$. If we take the maximal $\partial$-set of $\operatorname{DC}(n, a, b)\left[S_{6}\right]$ to the set $D_{9}$ having the set $D_{10}$, then since $\partial\left(K_{1, n}\right)=n-1$, we have $\partial\left(D_{10}\right)=\left\lfloor\frac{n-a-b}{3}\right\rfloor+(b-1)$. If we take the vertex $w_{1}$ of $\operatorname{DC}(n, a, b)$ to the set $D_{10}$ having the set $D_{11}$, we have $\partial\left(D_{11}\right)=\left\lfloor\frac{n-a-b}{3}\right\rfloor+(b+a-3)$, and taking any other subset of $V(\mathrm{DC}(n, a, b))$ to the set $D_{11}$ yields $\partial\left(D_{11}\right)<\left\lfloor\frac{n-a-b}{3}\right\rfloor+(b+a-3)$.

For $n-a-b=3 k$ or $n-a-b=3 k+2(k \in \mathbb{Z})$, if we take the maximal $\partial$-set of $P_{n-a-b}$ to the set $D_{12}$ such that $w_{1}, w_{n-a-b} \in B\left(D_{12}\right)$, then since $\partial\left(P_{n}\right)=\left\lfloor\frac{n}{3}\right\rfloor$, we have $\partial\left(D_{12}\right)=\left\lfloor\frac{n-a-b}{3}\right\rfloor$. If we take the vertex $w_{1} \in V(\mathrm{DC}(n, a, b))$ to the set $D_{12}$ having the set $D_{13}$, we have $\partial\left(D_{13}\right)=\left\lfloor\frac{n-a-b}{3}\right\rfloor+(b-2)$. If we take the vertex $w_{n-a-b} \in V(\mathrm{DC}(n, a, b))$ to the set $D_{13}$ having the set $D_{14}$, we have $\partial\left(D_{14}\right)=\left\lfloor\frac{n-a-b}{3}\right\rfloor+(b+a-4)$, and taking any subset of $V(\operatorname{DC}(n, a, b))$ to the set $D_{14}$ yields $\partial\left(D_{14}\right)<\left\lfloor\frac{n-a-b}{3}\right\rfloor+(b+a-4)$. If we take the vertex $w_{n-a-b} \in V(\mathrm{DC}(n, a, b))$ to the set $D_{12}$ having the set $D_{15}$, then we have $\partial\left(D_{15}\right)=\left\lfloor\frac{n-a-b}{3}\right\rfloor+(a-2)$.

By the definition of graph differential, we get

$$
\begin{aligned}
& \partial(\operatorname{DC}(n, a, b))=\max \left\{\partial\left(D_{l}\right)\right\} \quad(1 \leq l \leq 15) \\
& \partial(\operatorname{DC}(n, a, b))=a+b+\left\lfloor\frac{n-a-b-4}{3}\right\rfloor \text { for } a, b>1 \text { and } n-a-b>2 .
\end{aligned}
$$

Thus the proof holds.

Remark 5.4. We can easily observe that $\gamma(\mathrm{DC}(n, a, b))=2+\left\lceil\frac{n-a-b-4}{3}\right\rceil$ for $a, b>1$ and by Theorem 2.2 we conclude that double comets are dominant differential networks for $n-a-b=3 k$ or $n-a-b=3 k+1(k \in \mathbb{Z})$.

\subsection{Lollipop networks}

The lollipop network $L_{n, d}$ is a graph obtained from a complete graph $K_{n-d}$ and a path $P_{d}$, by joining one of the end vertices of $P_{d}$ [18], let this vertex be the vertex $u$, to all the vertices of $K_{n-d}$.

Theorem 5.5. The differential of the lollipop network $L_{n, d}(d>1)$ of order $n$ is

$$
\partial\left(L_{n, d}\right)=n-d+\left\lfloor\frac{d-2}{3}\right\rfloor .
$$

Proof. If we take the vertex $u$ of $L_{n, d}$ to the set $D_{1}$, that is $D_{1}=\{u\}$, then we have $\partial\left(D_{1}\right)=n-d$.

Let $S_{1}=V\left(L_{n, d}\right) \backslash N_{L_{n, d}}[u]$ and so we have that $L_{n, d}\left[S_{1}\right]=P_{d-2}$. If we take the maximal $\partial$-set of $L_{n, d}\left[S_{1}\right]$ to the set $D_{1}$ having the set $D_{2}$, then since $\partial\left(P_{n}\right)=\left\lfloor\frac{n}{3}\right\rfloor$, we have $\partial\left(D_{2}\right)=n-d+\left\lfloor\frac{d-2}{3}\right\rfloor$, and taking any other subset of $V\left(L_{n, d}\right)$ to the set $D_{2}$ yields $\partial\left(D_{2}\right)<n-d+\left\lfloor\frac{d-2}{3}\right\rfloor$. 
If we take one of the vertices of $K_{n-d}$ to the set $D_{3}$, then we have $\partial\left(D_{3}\right)=n-d-1$.

Let $S_{2}=V\left(L_{n, d}\right) \backslash N_{L_{n, d}}\left[D_{3}\right]$ and so we have that $L_{n, d}\left[S_{2}\right]=P_{d-1}$. If we take the maximal $\partial$-set of $L_{n, d}\left[S_{2}\right]$ to the set $D_{3}$ having the set $D_{4}$, then since $\partial\left(P_{n}\right)=\left\lfloor\frac{n}{3}\right\rfloor$, we have $\partial\left(D_{4}\right)=n-d-1+\left\lfloor\frac{d-1}{3}\right\rfloor$, and taking any other subset of $V\left(L_{n, d}\right)$ to the set $D_{4}$ yields $\partial\left(D_{4}\right)<n-d-1+\left\lfloor\frac{d-1}{3}\right\rfloor$.

If we take the maximal $\partial$-set of $P_{d}$ to the set $D_{5}$, such that $u \in B\left(D_{5}\right)$, then since $\partial\left(P_{n}\right)=\left\lfloor\frac{n}{3}\right\rfloor$, we have $\partial\left(D_{5}\right)=\left\lfloor\frac{d}{3}\right\rfloor$.

If we take the $\partial$-set of $K_{n-d}$ to the set $D_{5}$ having the set $D_{6}$, then since $\partial\left(K_{n}\right)=n-2$, we get $\partial\left(D_{6}\right)=\left\lfloor\frac{d}{3}\right\rfloor+n-d-2$ and taking any other subset of $V\left(L_{n, d}\right)$ to the set $D_{6}$ yields $\partial\left(D_{6}\right)<\left\lfloor\frac{d}{3}\right\rfloor+n-d-2$.

By the definition of graph differential, we get

$$
\begin{aligned}
& \partial\left(L_{n, d}\right)=\max \left\{\partial\left(D_{i}\right)\right\} \quad(1 \leq i \leq 6) \\
& \partial\left(L_{n, d}\right)=n-d+\left\lfloor\frac{d-2}{3}\right\rfloor \text { for } d>1
\end{aligned}
$$

Thus the proof holds.

Remark 5.6. We can easily observe that $\gamma\left(L_{n, d}\right)=1+\left\lceil\frac{d-2}{3}\right\rceil$ for $d>1$ and by Theorem 2.2 we conclude that lollipop networks are dominant differential for $d=3 k+2$ or $d=3 k+4(k \in \mathbb{Z})$.

\section{4. $E_{p}^{t}$ networks}

The network $E_{p}^{t}$ is a tree of order $p t+2$ obtained from a path with two vertices having one of the end-vertices attached to $t$ legs and each leg has $p$ vertices [10]. Let the end-vertex attached to $t$ legs be the vertex $c$, and the vertex degree of the vertex $c$ is $\operatorname{deg}(c)=t+1$.

Theorem 5.7. The differential of the $E_{p}^{t}$ network of order $p t+2$ is

$$
\partial\left(E_{p}^{t}\right)=t\left(\left\lfloor\frac{p-1}{3}\right\rfloor+1\right) .
$$

Proof. If we take the vertex $c$ of $E_{p}^{t}$ to the set $D_{1}$, then we have $\partial\left(D_{1}\right)=t$.

Let $S_{1}=V\left(E_{p}^{t}\right) \backslash N_{E_{p}^{t}}[c]$ and so we have that $E_{p}^{t}\left[S_{1}\right]=\bigcup_{i=1}^{t} P_{p-1}$. If we take the maximal $\partial$-set of $E_{p}^{t}\left[S_{1}\right]$ to the set $D_{1}$ having the set $D_{2}$, then since $\partial\left(P_{n}\right)=\left\lfloor\frac{n}{3}\right\rfloor$, we have $\partial\left(D_{2}\right)=t+t\left\lfloor\frac{p-1}{3}\right\rfloor$, and taking any other subset of $V\left(E_{p}^{t}\right)$ to the set $D_{2}$ yields $\partial\left(D_{2}\right)<t+t\left\lfloor\frac{p-1}{3}\right\rfloor$.

If we take the other end-vertex that is adjacent to the vertex $c$ in $P_{2}$ to the set $D_{3}$, then we have $\partial\left(D_{3}\right)=0$.

Let $S_{2}=V\left(E_{p}^{t}\right) \backslash N_{E_{p}^{t}}\left[D_{3}\right]$ and so we have that $E_{p}^{t}\left[S_{2}\right]=\bigcup_{i=1}^{t} P_{p}$. If we take the maximal $\partial$-set of $E_{p}^{t}\left[S_{2}\right]$ to the set $D_{3}$ having the set $D_{4}$, then since $\partial\left(P_{n}\right)=\left\lfloor\frac{n}{3}\right\rfloor$, we receive $\partial\left(D_{4}\right)=t\left\lfloor\frac{p}{3}\right\rfloor$, and taking any other subset of $V\left(E_{p}^{t}\right)$ to the set $D_{4}$ yields $\partial\left(D_{4}\right)<t\left\lfloor\frac{p}{3}\right\rfloor$.

If we take the maximal $\partial$-set of one of the legs to the set $D_{5}$ such that $c \in B\left(D_{5}\right)$, then since $\partial\left(P_{n}\right)=\left\lfloor\frac{n}{3}\right\rfloor$, we have $\partial\left(D_{5}\right)=\left\lfloor\frac{p+1}{3}\right\rfloor$.

If we take the maximal $\partial$-sets of $t-1$ legs of $E_{p}^{t}$ to the set $D_{5}$ having the set $D_{6}$, since $\partial\left(P_{n}\right)=\left\lfloor\frac{n}{3}\right\rfloor$, we receive $\partial\left(D_{6}\right)=\left\lfloor\frac{p+1}{3}\right\rfloor+(t-1)\left\lfloor\frac{p}{3}\right\rfloor$, and taking any other subset of $V\left(E_{p}^{t}\right)$ to the set $D_{6}$ yields $\partial\left(D_{6}\right)<t+t\left\lfloor\frac{p+1}{3}\right\rfloor$.

By the definition of graph differential, we get

$$
\begin{aligned}
& \partial\left(E_{p}^{t}\right)=\max \left\{\partial\left(D_{i}\right)\right\} \quad(1 \leq i \leq 6) \\
& \partial\left(E_{p}^{t}\right)=t\left(\left\lfloor\frac{p-1}{3}\right\rfloor+1\right)
\end{aligned}
$$

Thus the proof holds.

Remark 5.8. We can easily observe that $\gamma\left(E_{p}^{t}\right)=1+t\left\lceil\frac{p-1}{3}\right\rceil$ and by Theorem 2.2 we conclude that $E_{p}^{t}$ networks are dominant differential for $p=3 k$ or $p=3 k+1(k \in \mathbb{Z})$. 


\section{REFERENCES}

[1] L.A. Basilio, S. Bermudo and J.M. Sigarreta, Bounds on the differential of a graph. Utilitas Math. 103 (2017) $319-334$.

[2] S. Bermudo, On the differential and Roman domination number of a graph with minimum degree two. Disc. Appl. Math. 232 (2017) 64-72.

[3] S. Bermudo and H. Fernau, Lower bounds on the differential of a graph. Disc. Math. 312 (2012) 3236-3250.

[4] S. Bermudo and H. Fernau, Computing the differential of a graph: hardness, approximability and exact algorithms. Disc. Appl. Math. 165 (2014) 69-82.

[5] S. Bermudo and H. Fernau, Combinatorics for smaller kernels: the differential of a graph. Theor. Comput. Sci. 562 (2015) 330-345.

[6] S. Bermudo, H. Fernau and J.M. Sigarreta, The differential and the Roman domination number of a graph. Appl. Anal. Disc. Math. 8 (2014) 155-171.

[7] S. Bermudo, J.C. Hernández-Gómez, J.M. Rodríguez and J.M. Sigarreta, Relations between the differential and parameters in graphs. Electron. Notes Disc. Math. 46 (2014) 281-288.

[8] S. Bermudo, L. De la Torre, A.M. Martín-Caraballo and J.M. Sigarreta, The differential of the strong product graphs. Int. J. Comput. Math. 92 (2015) 1124-1134.

[9] S. Bermudo, J.M. Rodríguez and J.M. Sigarreta, On the differential in graphs. Utilitas Math. 97 (2015) $257-270$.

[10] T.H. Cormen, C.E. Leiserson, R.L. Rivest and C. Stein, Introduction to Algorithms, 3rd edition. The MIT Press, Cambridge, MA (2009).

[11] M. Cygan, M. Pilipczuk and R. Škrekovski, Relation between Randić index and average distance of trees. MATCH Commun. Math. Comput. Chem. 66 (2011) 605-612.

[12] J.A. Gallian, A dynamic survey of graph labeling. Electron. J. Comb. 15 (2008) DS6.

[13] J.H. Hattingh and E. Ungerer, The signed and minus $k$-subdomination numbers of comets. Disc. Math. 183 (1998) $141-152$.

[14] J.C. Hernández-Gómez, Differential and operations on graphs. Int. J. Math. Anal. 9 (2015) 341-349.

[15] I. Javaid and S. Shokat, On the partition dimension of some wheel related graphs. J. Prime Res. Math. 4 (2008) $154-164$.

[16] J.R. Lewis, Differentials of graphs, Master's thesis. East Tennessee State University, Johnson City, TN (2004).

[17] J.L. Mashburn, T.W. Haynes, S.M. Hedetniemi, S.T. Hedetniemi and P.J. Slater, Differentials in graphs. Utilitas Math. 69 (2006) 43-54.

[18] M.J. Morgan, S. Mukwembi and H.C. Swart, On the eccentric connectivity index of a graph. Disc. Math. 311 (2011) $1229-1234$.

[19] P.R.L. Pushpam and D. Yokesh, Differential in certain classes of graphs. Tamkang J. Math. 41 (2010) $129-138$.

[20] J.M. Sigarreta, Differential in cartesian product graphs. Ars Comb. CXXVI (2016) 259-267.

[21] D.B. West, Introduction to Graph Theory. Prentice Hall, NJ (2001). 